\title{
Essai sur le rôle d'une espèce végétale rustique pour un développement durable de la steppe algérienne
}

\section{Mohammedi Halima, Labani Abderrahmane et Benabdeli Khéloufi}

\section{Q OpenEdition}

Journals

Édition électronique

URL : http://journals.openedition.org/developpementdurable/2925

DOI : 10.4000/developpementdurable.2925

ISSN : 1772-9971

Éditeur

Association DD\&T

Référence électronique

Mohammedi Halima, Labani Abderrahmane et Benabdeli Khéloufi, «Essai sur le rôle d'une espèce végétale rustique pour un développement durable de la steppe algérienne », Développement durable et territoires [En ligne], Varia (2004-2010), mis en ligne le 17 juillet 2006, consulté le 01 mai 2019. URL : http://journals.openedition.org/developpementdurable/2925; DOI : 10.4000/ developpementdurable.2925

Ce document a été généré automatiquement le 1 mai 2019.

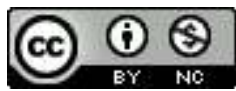

Développement Durable et Territoires est mis à disposition selon les termes de la licence Creative Commons Attribution - Pas d'Utilisation Commerciale 4.0 International. 


\title{
Essai sur le rôle d'une espèce végétale rustique pour un développement durable de la steppe algérienne
}

\author{
Mohammedi Halima, Labani Abderrahmane et Benabdeli Khéloufi
}

1 Le développement économique et social d'une région est subordonné à une gestion tant raisonnée que rationnelle de son environnement physique, biologique et socioéconomique. L'état actuel de dégradation atteint dans les zones arides steppiques représentant plus de 36 millions d'hectares est un problème majeur pour l'Algérie du $3^{\text {zème }}$ millénaire (Benabdeli, 1992). D'énormes potentialités en termes de ressources naturelles risquent d'être irréversiblement compromises par l'évolution du climat et les mutations socio-économiques dans le milieu steppique qui reste l'ultime barrière naturelle contre le désert. Cet espace occupe plus de 20 millions d'hectares dans le Tell algérien et est sérieusement menacé par le processus de désertification.

De nombreux spécialistes et une multitude de séminaires n'ont cessé de souligner la gravité et l'aggravation constante des phénomènes de dégradation de cet espace et l'urgence d'adopter les solutions adéquates afin d'y remédier. Les quelques tentatives dans ce domaine ont pratiquement échoué et les résultats obtenus sont très loin des espoirs escomptés, malgré les sommes investies.

3 En l'absence d'investigation écologique et socio-économique en adéquation avec le milieu humain et physico-biologique, il reste utopique de prétendre protéger cette zone stratégique. Les nombreux travaux effectués depuis près de quatre décennies ont mis en relief une dégradation progressive de la couverture végétale de la steppe et $\mathrm{du}$ sol. Comme conséquence directe de cette situation, une désertification du milieu s'installe et se développe.

4 Identifier les principales causes de l'échec du développement durable de la steppe constitue le but de ce travail, le second objectif est d'apprécier l'impact phyto-écologique de l'introduction d'une espèce rustique, l'Ailanthus glandulosa Desf., qui présente des 
caractéristiques très intéressantes, comme l'adaptation aux conditions du climat, du sol et au contexte social de la zone.

5 L'analyse des causes de l'échec des plans de développement de la steppe algérienne et du rôle de l'espèce végétale qu'est Ailanthus glandulosa Desf. pour un développement durable repose sur les étapes suivantes :

- tout d'abord nous présentons quelques généralités sur les aspects socio-économiques et écologiques de la steppe

- ensuite nous nous penchons sur les échecs des stratégies successives et l'analyse des causes de ces échecs

- enfin nous abordons l'impact de écologique et socio-économique de l'introduction d' Ailanthus glandulosa.

6 La Steppe algérienne constitue une vaste région qui s'étend du Sud de l'Atlas saharien, formant un ruban de $1000 \mathrm{~km}$ de long sur $300 \mathrm{~km}$ de large, réduite a moins de $150 \mathrm{~km}$ à l'Est. Elle s'étend sur une superficie de 36 millions d'hectares mais compte 20 millions d'hectares de parcours et sa limite Nord commence avec le tracé de la limite des précipitations moyennes annuelles de $400 \mathrm{~mm}$, pour se limiter au Sud de l'Atlas saharien à $100 \mathrm{~mm}$ de précipitations.

Figure 1 : Localisation de la steppe en Algérie

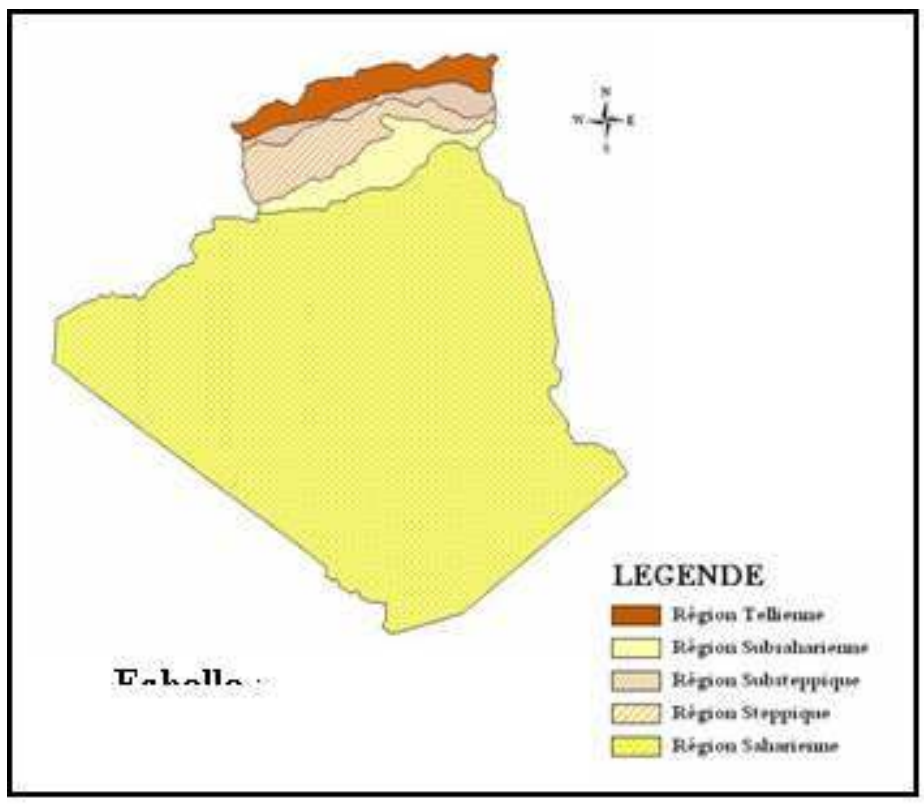

7 La steppe se caractérise par un climat semi-aride sur sa partie Nord et un climat aride sur sa frange Sud. Les précipitations moyennes sont comprises entre les isohyètes 400 et 100 $\mathrm{mm}$, tranche insuffisante pour permettre une utilisation agricole intensive. En année sèche, l'ensemble de la steppe reçoit moins de $250 \mathrm{~mm}$ de pluie; cet isohyète remonte jusqu'au piémont du Tell.

La végétation steppique est une végétation basse et discontinue, composée de plantes herbacées, généralement en touffes, laissant apparaître entre elles des plaques de sol nu. Cette végétation est diversifiée par sa composition floristique et sa densité, et reste l'enjeu fondamental de la vie du pasteur. C'est elle qui détermine les déplacements de ce dernier et ses lieux de stationnement avec ses troupeaux. 
Quatre zones peuvent être distinguées dans l'espace steppique :

- Zone 1 : avec une superficie de 700.000 à 1.000 .000 d'hectares, elle reçoit des précipitations annuelles moyennes de $400 \mathrm{~mm}$, c'est essentiellement le domaine des maquis, des garrigues et des forêts de l'Atlas saharien.

- Zone 2 : occupant entre 3,5 et 4.000 .000 d'hectares, elle bénéficie d'une pluviométrie comprise entre 300 et $400 \mathrm{~mm}$. Il s'agit de la zone steppique la plus favorisée, située sur la frange nord de l'Atlas tellien. C'est la zone des « Parcours vrais » qui supporte une importante charge pastorale (2 à 4 moutons à l'hectare). Elle est cependant occupée par la céréaliculture rentable en saison pluvieuse.

- Zone 3 : elle couvre entre 5 et 6.000 .000 d'hectares et connaît des précipitations moyennes annuelles comprises entre 200 et $300 \mathrm{~mm}$. C'est la région des hautes plaines centrales et méridionales et le versant Sud de l'Atlas saharien. Les parcours sont de qualité moyenne et la charge pastorale y est plus faible (2 moutons à l'hectare).

- Zone 4 : elle s'étend sur 10.000.000 hectares avec une pluviométrie annuelle très limitée, entre 100 et $200 \mathrm{~mm}$, c'est la région sud du Hodna et le piémont sud atlasique. La charge pastorale y est très faible.

10 Les surfaces offrant une biomasse intéressante pour les troupeaux sont donc théoriquement importantes, si l'on exclut 5.000.000 d'hectares occupés par les cultures, les forêts et les zones improductives, elles totaliseraient 15.000.000 d'hectares. Toutefois, les potentialités réelles ne représentent, en réalité, que la moitié de cette superficie, en raison des immenses aires abandonnées, faute de points d'eau.

11 La Steppe est essentiellement composée d'une strate herbacée assez variée d'espèces vivaces et éphémères. Trois espèces y dominent traditionnellement la flore, à savoir l'Alfa (Stipa tenacissima L.), l'Armoise (Artemisia herba alba L.) et la fausse alfa (Lygeum spartum). Plus d'une trentaine d'autres espèces y végètent à différentes périodes de l'année. L'Alfa et l'Armoise occupent à elles seules près de 7.000.000 d'hectares tandis que le Lygeum occupe 3.000.000 d'hectares. Généralement, de nombreuses espèces halophiles occupent des sols salins aux alentours des chotts (Djebaili, 1984). Les surfaces pastorales offrent une diversité alimentaire appréciable, eu égard à la diversité des espèces et leur qualité ainsi qu'à la période où chaque plante est consommable par le cheptel.

Le cheptel ovin national est le premier fournisseur de viande rouge, soit 68.000 tonnes en 1983. En 1996, il fournissait $75 \%$ des 2.996 .000 quintaux produits. Sa contribution à l'économie nationale est importante dans la mesure où il représente un capital de plus de 1.000.000.000 de dinars. En 1985, sur un effectif national de 15.500.000 têtes, quelques 11.500.000 têtes restaient cantonnées dans la steppe. En 1996 l'effectif du cheptel ovin s'élevait à 17.301 .000 têtes dont 75\% concentrées dans la zone steppique (Bouchetata, 2002).

14 Avec les possibilités d'accès à la propriété foncière, les superficies emblavées en céréaliculture éphémère se développent ainsi que les petites agglomérations. Le défrichement avec des moyens mécaniques importants constitue un nouveau modèle d'utilisation de la steppe par les éleveurs, ils ne sont plus pasteurs mais des agro-éleveurs occasionnels. L'évolution du système d'élevage découlant de l'aide apportée par l'Etat aux propriétaires (subventions, terres, moyens matériels) s'est traduite par un début de sédentarisation. Cette dernière se fait de plus en plus sentir et son impact sur l'espace steppique est de plus en plus dégradant. L'augmentation de l'effectif des troupeaux et la 
facilité de le déplacer avec des camions à la recherche d'herbe constituent d'autres facteurs augmentant la pression sur la steppe.

Une steppe aride est un milieu qui, par définition, n'offre que des conditions extrêmes pour l'établissement et le maintien d'une végétation pérenne. C'est cette végétation pérenne, qui joue un rôle fondamental dans la structure et le fonctionnement de l'écosystème, qui doit être réhabilitée. Malgré tous les programmes de développement appliqués à la zone steppique, cet espace reste sujet à la dégradation et au très faible taux de réussite des plantations végétales. Les conséquences qui en découlent sont alarmantes et se traduisent essentiellement par une altération des qualités physico-chimiques des sols, une érosion intense et une raréfaction du potentiel biologique.

Selon Floret et al (1981), le couvert végétal naturel y est soumis en permanence à un double impact, celui des sols (trop secs et légers) et du climat (faibles précipitations) d'une part et anthropogène (action de l'homme et de l'animal) d'autre part.

Le milieu steppique a été l'objet de plusieurs projets de développement depuis 1962 qui peuvent se classer en trois catégories de démarche aussi médiocres les unes que les autres.

Cette période se distingue par trois phases signifiantes :

- De 1962 à 1970 : l'Etat a tenté d'organiser les populations pastorales en les groupant dans des coopératives sur des territoires bien délimités, l'objectif était d'organiser ces populations pour permettre une exploitation rationnelle des parcours. Le nombre de coopératives créées était très faible par rapport à la population autochtone, les zones délimitées n'étaient pas en adéquation avec les habitudes des éleveurs et le système de vie de ces habitants a été bouleversé. Cela s'est traduit par un abandon de cette politique et une disparition pure et simple de ces coopératives.

- De 1970 à 1980 : cette période a connu la promulgation de la charte portant révolution agraire notamment le code pastoral. Son objectif était l'aménagement intégré de l'espace et la transformation radicale des rapports sociaux et du système de production, sans une connaissance approfondie de leurs systèmes tant social que productif. Cette période se distingue par trois faits importants mais sans apport positif pour l'espace steppique.

- La révolution agraire : avec une stratégie basée sur l'organisation des agro-pasteurs en coopératives dont l'impact, tant sur le changement du comportement des éleveurs que sur la sédentarisation des populations, a été négatif. Cette phase n'a finalement touché qu'à peine $3 \%$ des éleveurs de toute la région et uniquement $5 \%$ du cheptel de la steppe. L'Etat a aussi soutenu l'alimentation des ovins par l'importation et la distribution d'orge dont les conséquences ont été catastrophiques sur les systèmes d'élevage traditionnels.

19 L'objectif essentiel de ce projet était la lutte contre la désertification par le boisement et le reboisement sur plus de 3 millions d'hectares avec introduction de l'arboriculture rustique et d'espèces fourragères également. Toutes les opérations entamées ont été faites sans études au préalable et les résultats obtenus n'étaient pas satisfaisants dans leur ensemble. Parmi les causes principales de cet échec, il faut noter le mauvais choix des espèces végétales et des techniques de plantation, la transformation d'un espace de parcours en zone boisée, l'absence d'intégration de la population dans le projet et l'inadéquation entre la vocation des terres et l'aménagement réalisé.

La création du Secrétariat d'Etat aux forêts et au reboisement en 1980 a permis d'apporter des correctifs en cessant les reboisements souvent improvisés. Une politique de classification des terres et l'élaboration de schémas directeurs d'aménagement des zones 
pilotes ainsi que la reconstitution des massifs forestiers dégradés furent les principales actions engagées. D'autres actions ont pu être entreprises telles que les plantations pastorales, la fixation des dunes, les ouvertures de piste. Ces actions sont restées modestes par rapport à l'importance de la surface de la zone steppique estimée à 20 millions d'hectares.

De 1980 à 1996: cette période correspond à une nouvelle orientation de la politique agricole du pays avec la promulgation de quatre lois: celle portant accession à la propriété foncière agricole par la mise en valeur en 1983, la loi portant mode exploitation des terres agricoles en 1987, la loi portant orientation foncière en 1990 et la loi portant intégration dans le domaine privé de l'Etat des terres pastorales et à vocation pastorale à une réglementation spéciale de protection, de gestion et d'exploitation conformément au code pastoral. La création d'un organisme chargé de la gestion de la steppe en 1985 constitue un autre fait marquant de cette période, cette institution devait maîtriser le milieu et prendre en charge les programmes d'aménagement des parcours steppiques. Durant cette période plus de 156000 ha ont été attribués à 23700 acquéreurs, soit en moyenne des exploitations de 6 ha, un morcellement de l'espace productif entravant toute politique d'aménagement global de cette entité écologique et géographique (Bedrani, 1996). De l'avis de plusieurs auteurs spécialistes de la steppe, il ressort que les causes profondes et déterminantes de l'échec des différentes actions menées sont dues au statut juridique des terres en général et de la steppe en particulier.

Cette période se caractérise par des objectifs d'aménagement, de régulation du cheptel, de développement agricole et forestier. On y distingue trois approches :

- l'approche aménagement : L'une de ses principales mesures est de permettre une répartition équilibrée du peuplement et des activités par un développement global et durable. Il s'agit de déceler les déséquilibres existant en terme de couverture des besoins essentiels des populations et de provoquer l'émergence des centres à promouvoir. Ce programme implique de nombreux investissements, ce qui demande des disponibilités financières importantes difficiles à réunir dans le contexte économique.

- l'approche par régulation du cheptel : Aucune des mesures prises dans ce volet (impôts, révision des coûts de production, exclusivité du droit d'usage des terres steppiques...) n'a permis de réguler l'effectif des troupeaux selon les possibilités offertes par l'espace steppique, entraînant une surcharge pastorale avec toutes ses conséquences sur le milieu physique et biologique.

l'approche du secteur de l'agriculture et des forêts : Cette approche a fait surtout appel à des actions purement techniques d'amélioration pastorale, de mise en défens, de plantation d'arbustes fourragères, de mise en place d'ouvrages anti-érosifs, de correctifs apporté au barrage vert par la diversification des espèces (Nedjahi, 1998).

Pourquoi tous les programmes et toutes les approches en matière d'aménagement et de développement de l'espace steppique se sont-ils avérés vains? C'est une question à laquelle il faut répondre pour identifier les vraies causes de ces échecs et asseoir une politique de développement basée sur une stratégie globale.

Dans un premier temps, tous les efforts consentis dans cet espace ont été trop polarisés sur le pastoralisme, l'élevage du mouton, l'exploitation de l'alfa, alors que ces richesses sont limitées: l'alfa est une matière première aux volumes de production limités (régénération naturelle très aléatoire), et l'élevage du mouton subit les effets de la sécheresse dévastatrice qui rend toute production aléatoire en milieu aride. 
26 Il était suicidaire de baser toute l'économie de ce vaste territoire naturel de plus de 20 millions d'hectares sur l'exploitation exclusive de ces seules ressources. Même en surestimant le produit de vente de l'alfa, les apports de l'élevage du mouton, il était inconcevable de développer cet espace sans des investissements dans les autres activités économiques tant agricoles qu'industrielles en apport avec la production de la steppe.

Dans un deuxième temps, le secteur agricole avec l'aspect pastoral, agro-pastoral et agrosylvo-pastoral allait être considéré comme un vecteur de développement devant servir de support économique à la vie des populations locales. Or les terres agricoles dans cet espace n'étaient pas excédentaires et pourtant de nouveaux agriculteurs attirés par la disponibilité des ressources en eau se sont installés. Les réserves en eau souterraines sont estimées à plus de 60 milliards de $\mathrm{m}^{3}$ et leur taux d'exploitation n'est que de $20 \%$ alors qu'il est de $90 \%$ dans le nord du pays. Les possibilités d'exploitation annuelle des ressources en eau souterraine de la zone steppique sont évaluées à 1 milliard de $\mathrm{m}^{3}$ (Benabdeli, 1995). L'activité agricole s'est développée sans relation avec l'activité pastorale, et aucun investissement garantissant un développement durable n'a été réalisé.

Une prédominance de l'approche sectorielle aggravée par une vision disciplinaire étroite à l'intérieur de chaque secteur est à l'origine des échecs de préservation de la steppe. Pour les écologues, phytosociologues et agronomes spécialisés en phytotechnie, l'essentiel est le pâturage steppique. Leur priorité était de dresser des cartes des potentialités sans pour autant finaliser la stratégie d'exploitation et de préservation des ressources. L'introduction d'espèces herbacées exotiques (Cactus, Atriplex, Médicago) n'a donné aucun résultat encourageant.

)Pour les zootechniciens, l'avenir était à l'introduction de races ovines ou caprines prolifiques ou ayant des aptitudes particulières pour la production de viande, de lait, de laine. C'est ainsi qu'il y eut plusieurs introductions à la mode : chèvre alpine chamoisée, mouton Romanov et Merinos...et pour les partisans des races locales, les avis étaient partagés sur les valeurs respectives de chacune : Demen, Hamra, Ouled Djellal...

30 «Il est normal que des hommes ayant une formation de base et de spécialités différentes expriment des points de vue différents concernant un problème donné mais un consensus technique et socio-économique doit être trouvé pour préserver et développer durablement cet espace stratégique » notait Benabdeli (1992).

31 En l'espace de moins d'un demi siècle les modes d'exploitation de la steppe ont été totalement bouleversés tant par les décisions politiques que les approches techniques. Dans ce volet on distingue le système traditionnel et le système moderne, chacun avec ses avantages et ses inconvénients, et leur impact sur le développement durable de la steppe. 3.2.1 Le système

Le système traditionnel d'élevage et de gestion des parcours steppiques, qui garantissait un équilibre biologique avec une surveillance de l'effectif du cheptel excluait tout surpâturage. Les facteurs environnementaux locaux (manque d'eau, distance à parcourir, absence de soins vétérinaires, manque de moyens de transport) participaient à réguler la taille des troupeaux selon les potentialités des espaces. La reproduction du cheptel restait traditionnelle et naturelle et demandait quelques années, laissant le temps aux parcours de se régénérer à la faveur de périodes humides, l'équilibre biologique était alors progressivement rétabli. 

changer fondamentalement les données du problème pastoral et bouleverser l'équilibre précaire de la zone steppique. Plusieurs éléments nouveaux et complémentaires sont intervenus et ont provoqué la rupture de cet équilibre. Les principales causes sont :

- La modernisation des systèmes d'élevage ovin : le productivisme allait faire évoluer rapidement les méthodes et les équipements concernant les principaux facteurs de production que sont l'alimentation, l'habitat, l'abreuvement, les transports, les soins vétérinaires. L'apport d'alimentation, facteur d'amélioration déterminant, a été à l'origine de l'engraissement « in situ » se traduisant par la rupture de l'équilibre cheptel/pâturage à cause de la forte charge pastorale. Les aliments étaient acquis directement auprès des opérateurs publics car subventionnés. Il s'agit des céréales, son, fourrage sec, paille et des aliments composés complémentaires des fourrages secs. L'abreuvement du cheptel, qui était un autre facteur limitant le développement des troupeaux, accentué par le contexte climatique des parcours steppiques, a connu une issue positive par le forage de puits totalement équipés pour les villages nouvellement installés dans les hauts Plateaux.

- Les activités de transport : les moyens de transport de l'eau furent renforcés par l'acquisition de moyens pour le transport du cheptel et des aliments. Remorques, camions, bétaillères facilitaient l'approvisionnement et permettaient des déplacements rapides du cheptel vers chaque zone de pacage repérée, et cela, pour les zones menacées de surpâturage, correspondait à une augmentation de la capacité de nuisance du cheptel.

- Les progrès des soins vétérinaires : en matière de vêlage, ceux-ci ont eu comme impact direct un accroissement rapide des troupeaux. Tous les actes étaient supportés par l'Etat, comme les vaccinations réalisées dans le cadre de campagnes organisées pour l'application de programmes prophylactiques, qui assuraient une bonne maîtrise épidémiologique. Les produits vétérinaires étaient disponibles et cédés à des prix abordables.

- La sédentarisation de plus en plus importante, découlant de l'implantation de villages et de zones de vie, dont la conséquence est une perturbation des coutumes des habitants de l'espace steppique. Les systèmes d'élevage ne sont plus fondés sur la mobilité saisonnière et spatiale : si auparavant l'animal allait vers l'aliment, aujourd'hui c'est l'aliment qui va vers l'animal. La mécanisation est une des principales causes de la sédentarisation avec toutes ses conséquences négatives sur les écosystèmes steppiques, comme le défrichement pour la culture de céréales, le surpâturage imposé par l'augmentation des effectifs, les incendies pour la régénération des jeunes pousses d'alfa, l'éradication des espèces ligneuses pour le chauffage et la cuisine.

L'ensemble de ces progrès s'est traduit par une augmentation des effectifs du cheptel ovin évaluée entre 1970 et 2000 à 75\%, soit un taux d'accroissement annuel de $2.5 \%$. Les 
répercussions de cette constante augmentation du cheptel sont catastrophiques dans un premier temps sur la pérennité des parcours et dans un second temps sur l'équilibre de toute la région déjà menacée par le phénomène de la désertification. Il s'agit d'une réelle crise du pastoralisme, puisque pendant des siècles, les sociétés agro-pastorales étaient un exemple reconnu d'équilibre entre l'homme et le milieu naturel. Le principe de base de l'équilibre agro-pastoral est la mobilité. Les sociétés pastorales étaient nomades ou seminomades, pratiquant la transhumance. La règle était de ne jamais rester trop longtemps au même endroit. La pression sur le milieu était donc répartie dans le temps et dans l'espace, au rythme des saisons selon des règles précises et adoptées par tous les éleveurs. Ce système fonctionnait avec une organisation et de institutions tribales et un droit coutumier reconnu et respecté de tous. Malheureusement ces systèmes agro-pastoraux connaissent, depuis quelques décennies, des mutations profondes en raison d'une désorganisation de la société pastorale avec des erreurs de politique économique.

Pour ce qui est du foncier, à l'origine la terre appartenait à des collectivités qui vivaient en communautés et les exploitaient collégialement. De nos jours, les terres sont statutairement restées collectives, ou sont devenues propriété de l'Etat avec un droit de jouissance aux pasteurs. Or, la notion de communauté s'est considérablement amenuisée. Les liens tribaux et familiaux ne sont pas les mêmes. Le mode d'exploitation de la terre obéit à des règles de rentabilité, de spéculation, pour atteindre des objectifs individuels.

Comme la terre appartient à tout le monde, elle est exploitée anarchiquement et il n'y a plus de lien entre l'exploitant et la terre. Finalement, personne ne veut investir dans la préservation de l'écosystème ni même accepter d'imposer la discipline qui exige une gestion rationnelle (Bedrani, 1995).

La question foncière reste très complexe et ne peut trouver sa solution que dans une analyse politique, sociale et économique globale. Beaucoup de questions se posent comme :

- Que faire des ayants droits qui n'ont pas de troupeaux?

- Ne risque-t-on pas de morceler à outrance les parcours et de rendre la transhumance impossible?

- Que faire du droit coutumier qui reconnaît plus ou moins le droit de propriété à celui qui exploite sans interruption une parcelle?

- Que faire des éleveurs urbains et périurbains actuels?

Les enjeux sont importants et des conflits déjà chroniques existent, l'ensemble des problèmes évoqués concourent ainsi à exercer de fortes pressions sur des milieux déjà fragiles.

De l'analyse qui précède, il ressort que c'est l'élevage qui constitue l'élément déterminant dans l'économie et la dégradation de la steppe. Pour concilier la préservation des potentialités écologiques de la steppe et son exploitation par les pasteurs, le recours au développement durable peut être axé dans un premier temps sur l'introduction d'une espèce végétale, l'Ailanthus glandulosa Desf. Cette espèce a un impact certain sur le processus de dégradation observé dont le facteur principal est le parcours, elle permet avec son action écologique d'améliorer la productivité au niveau de la strate herbacée et agit positivement sur la préservation de la végétation et du milieu en général.

Actuellement, avec les possibilités rapides de déplacement des troupeaux par camions, tout l'espace steppique est concerné par une surexploitation. Le cheptel surtout ovin a augmenté en l'espace de 10 ans de plus de 3 millions et atteint actuellement 15 millions, 
accentuant la pression sur cet espace déjà fragilisé (Institut National de la Recherche Agronomique, 2002).

Ailanthus glandulosa quantifiés reposent sur les éléments suivants :

- une graine est considérée comme germée lorsque la radicule a percé les enveloppes.

- la précocité de la germination qui est exprimée par le taux des premières graines germées, après l'ensemencement.

- la vitesse de germination qui est le temps nécessaire pour avoir 50\% de semences germées après la date de semis.

- la capacité germinative qui est le pourcentage maximal de germination obtenu dans les conditions choisies par l'expérimentateur.

- le temps de latence qui est le temps mis pour avoir les premières graines germées (Mazliak, 1974).

Tous les auteurs ayant étudié cette espèce s'accordent à dire que Ailanthus glandulosa est une espèce peu exigeante en facteur du milieu (sol et climat) dotée d'une faculté de s'adapter aux différents types de sols et de bioclimat (subhumide, semi aride et aride). ${ }^{1}$ 


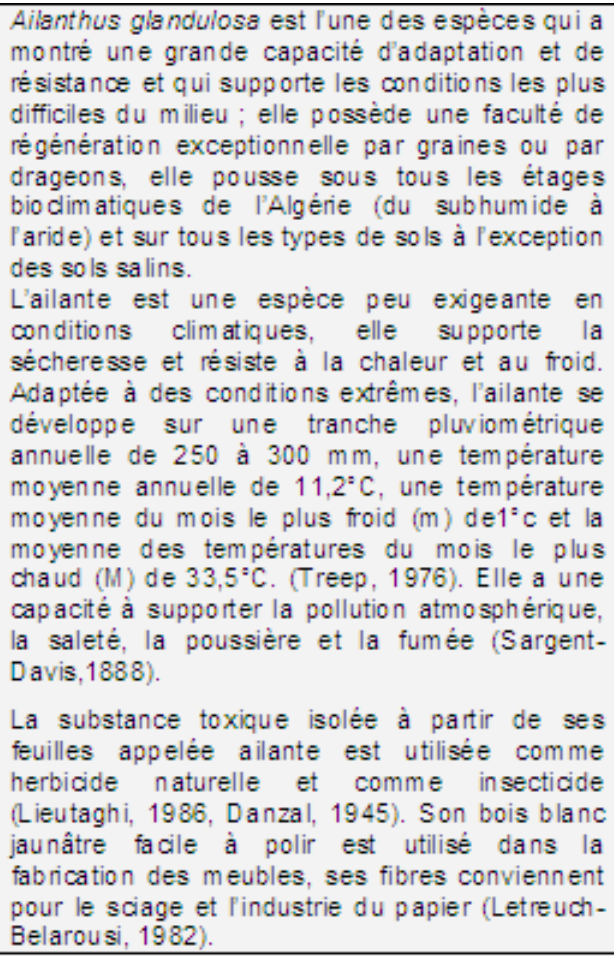

Les résultats obtenus (voir encadré 2) confirment que l'Ailanthus glandulosa trouve un biotope qui lui est favorable dans la zone steppique. ${ }^{2}$

51 Au niveau des bandes où a été planté Ailanthus glandulosa, une amélioration très perceptible de la phytomasse a été observée chez pratiquement toutes les espèces composant le cortège floristique de la steppe dans les différentes stations. L'amélioration moyenne toutes espèces confondues est de $83 \mathrm{~kg}$ de matière sèche par hectare. La biomasse verte induite par l'impact de l'Ailante est de l'ordre de $250 \mathrm{~kg}$, de quoi répondre aux besoins d'un ovin par hectare. Il s'en suivrait une diminution de la charge pastorale et par conséquent du surpâturage avec ses effets dévastateurs sur la végétation de la steppe.

Les résultats obtenus en matière de germination et de développement de l'espèce dans des conditions de milieu naturelles à la steppe permettent d'explorer plusieurs voies. La première serait de quadriller le territoire de la steppe par un réseau de plants d'ailante. L'impact écologique et socio-économique est certain et se résume à une amélioration de la productivité des parcours de plus de 100 unités fourragères par hectare, ce qui n'est pas négligeable en zone aride. Cette biomasse verte constituant une nourriture d'appoint au cheptel ovin est estimée à environ 1 milliard d'unités fourragères, soit de quoi répondre aux besoins alimentaires d'un cheptel de 2 millions de têtes. Cette productivité est induite par l'ombrage, la mobilisation de l'eau, la lutte contre le vent et la stabilisation $\mathrm{du}$ sol qu'apporte la plantation. Cette disponibilité fourragère agira sur le surpâturage en diminuant la pression animale d'un ovin par hectare.

Encadré 2 : Résultats des essais 


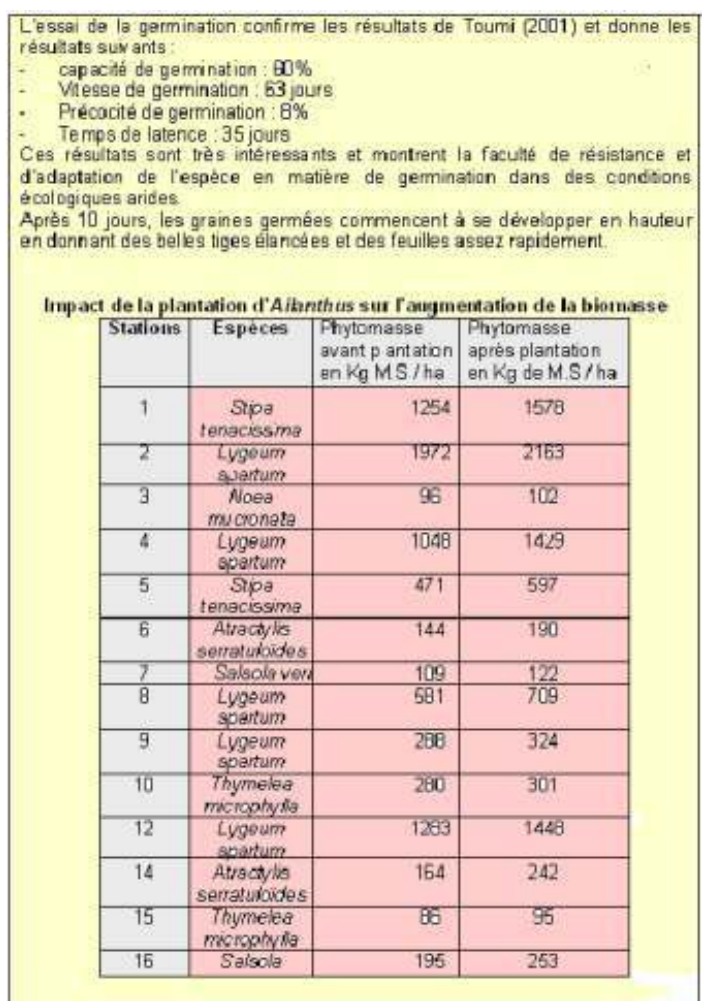

Avec un programme échelonné sur 10 ans et basé sur une plantation en quadrillage avec une densité de seulement 100 plants par hectare, les besoins en plants, pour les 10 millions d'hectare de la steppe les plus menacés, seraient de 100 millions de plants d' Ailanthus glandulosa annuellement. Le taux de boisement serait alors de $10 \%$ avec un impact tant écologique, social qu'économique.

Le nombre d'emplois directs découlant de la production annuelle de 100 millions de plants, de l'occupation d'une main d'œuvre pour la plantation et pour l'entretien, serait de plus de 16000 emplois permanents et plus de 4000 emplois saisonniers (le quart des emplois permanents, norme retenue dans les travaux de plantation). En effet nous estimons (Benabdeli, 2002) que l'introduction d'Ailanthus glandulosa nécessite 5000 journées de travail pour produire 1 million de plants, soit 250 jours de travail par an pour un emploi permanent.

En créant des emplois pour 20000 personnes en moyenne annuellement et sur une période de 10 ans, il est possible d'entamer la mise en place d'une approche axée sur le développement durable de la steppe par zone. Cette approche reposera sur une prise en charge de la population locale (éleveurs surtout) à travers la création d'emplois, base d'un revenu appréciable. 
Tableau ${ }^{\circ} 1$ : emplois générés par l'introduction d'Ailanthus glandulosa

\begin{tabular}{|c|c|c|c|}
\hline Action & $\begin{array}{l}\text { Normes } \\
\text { reten ues }\end{array}$ & $\begin{array}{l}\text { Journées de travail } \\
\text { pour } 1 \text { million } \\
\text { dhectare }\end{array}$ & $\begin{array}{l}\text { Emplois } \\
\text { créex } \\
\text { annuellemen }\end{array}$ \\
\hline $\begin{array}{l}\text { Production } \\
\text { deplant }\end{array}$ & $\begin{array}{l}10 \text { 000 } \\
\text { journées' } \\
\text { million de } \\
\text { plants } \\
50 \text { plante } \\
\text { parjour }\end{array}$ & 2000000 journées & 8000 \\
\hline $\begin{array}{l}\text { Entretien } \\
\text { plantation }\end{array}$ & $\begin{array}{l}100 \text { planta } \\
\text { par jour }\end{array}$ & 1000000 journées & 4000 \\
\hline $\begin{array}{l}\text { Emplos } \\
\text { indirects }\end{array}$ & & & 4000 \\
\hline Total & & 4000 000 iournées & 20000 \\
\hline
\end{tabular}

Source : Benabdeli, 2002

Les principales erreurs commises selon nous sont une absence de vision économique globale intégrant l'aménagement du territoire et de développement durable de cet espace, la prédominance de l'approche sectorielle, le changement brutal de système d'exploitation qui a provoqué la rupture de l'équilibre parcours/cheptel, la sédentarisation et la diminution des transhumances (Azzaba et de Achaba) qui ont aggravé la surexploitation des parcours par le surpâturage. Un autre volet important a été négligé dans l'approche de développement durable de la steppe, celui de la non prise en charge du comportement de la population locale et de ses principales activités dans l'espace steppique.

L'impact socio-économique des plantations à base d'Ailanthus glandulosa sur la zone sera appréciable puisque la production de biomasse verte sera multipliée presque par deux, et il sera possible d'augmenter la charge pastorale d'un ovin par hectare à deux sans perturber l'écosystème.

actuellement. Ce développement sera durable car il reposera sur une approche d'utilisation de cet espace sans l'altération de ses potentialités écologiques, en créant de l'emploi et en mettant à la disposition de la population de la nourriture pour les troupeaux.

courant et cause des dégâts importants sur la végétation dont le rôle est capital en zone aride. L'introduction de rangées d'Ailanthus glandulosa serait au bout de la 5 ème année un réservoir de prélèvement de bois pour les éleveurs (cuisson, chauffage, haie, enclos pour les moutons). Pour chaque hectare (100 plants), il est possible de prélever annuellement 20 stères de bois de chauffage, de quoi répondre aux besoins d'une famille.

steppique constituent les deux facteurs entravant tout développement durable de la steppe. Néanmoins le facteur anthropique est déterminant à plusieurs niveaux :

- au niveau des éleveurs, exploitants des parcours qui ont privilégié le profit à l'impératif de protection du milieu. La mécanisation et la motorisation ont donné à ces exploitants les moyens d'agresser les parcours de plus en plus fortement et sur des étendues de plus en plus grandes.

- au niveau des décideurs qui ont eu recours à des stratégies ayant largement fait la preuve de leurs limites. 
61 Pour faire face à cette situation, l'utilisation d'une espèce végétale pérenne à large spectre écologique qu'est l'ailante (Ailanthus glandulosa Desf.) permettrait d'initier le concept de développement durable. Ses qualités physiologiques et écologiques peuvent jouer un rôle très intéressant pour juguler le processus de dégradation de la steppe.

Elle constitue comme nous l'avons souligné un déterminant pour la durabilité de cet espace fragilisé tant par les conditions naturelles que les facteurs anthropiques (parcours, défrichement, incendies, labours). Les résultats préliminaires obtenus (germination et développement) permettent de préserver le sol, retenir l'humidité et améliorer la biomasse de la strate herbacée. En introduisant cette espèce, la pression animale sera limitée sur les espèces végétales de la steppe ${ }^{3}$. Elle peut être également source d'emplois et de combustible, et l'impact qu'elle aura sur la population contribuera à préserver et protéger celle-ci dans le cadre d'un programme de développement durable de cet espace.

\section{BIBLIOGRAPHIE}

Benbdei K., 1992, Aridité et production agricole. Journées scientifiques 1992 Inst. Sciences de la Nature, Université de Sidi Bel Abbes.

BENABDELI K ., 1995, Environnement écologique et gestion intégrée des ressources hydriques. 2ème Colloque Climat-environnement. Oran 24-25 Décembre 1995.

Benqbdeli K., 2002, Politique de création d'emplois verts en Algérie ; rapport M.A.T.E, 18 p.

Bdrani S., 1996, Foncier et gestion des ressources naturelles en Afrique du ord. Le cas de l'Algérie. O.S.S. 1-46 p.

Bedrani S., 1995, Les politiques agricoles et la dynamique des systèmes agropastoraux au Maghreb. Options Méditerranéennes, série B/n¹4 : 139-165.

Boucheta T., 2002, Diagnostic écologique et désertification, analyse des stratégies du milieu steppique. Magister Ecobiologie, C.U. Mascara.

Danzal M., 1974, Flore antiparasitaire pharmaceutique illustrée.

Djebaili S., 1983, Carte de l'occupation des terres de l'ALGERIE - Cartes pastorales de l'Algérie. Biocénoses $2(1,2,22-24)$.

Djebaili S., 1984, Steppe algérienne phytosociologie et écologie. O.P.U. 177

Floret C. et al, 1981, Dynamique des systèmes écologiques de la zone aride (application à l'aménagement sur des bases écologiques d'une zone de la Tunisie pré-saharienne)- Rapport, CEPE, Montpellier, $120 \mathrm{p}$.

I.N.R.A.A., 2002, Actes des quatrièmes journées scientifiques de l'I.N.R.A.A. Alger, 227 p.

Lieutaghi P., 1986, Le livre des arbres, arbustes et arbrisseaux. 354 p.

Letreuch-Belarocui N., 1982, Les reboisements en Algérie et leurs perspectives d'avenir. OPU vol. 1 et $2: 641 \mathrm{p}$.

Mazliak P., 1974, Physiologie végétale, croissance et développement. Paris 465 p. 
NEDJAHI A et al., 1998, Bilan des travaux de recherche sur la zone steppique. INRF, Alger .19 p.

Toumi F., 2001, Etude géobotanique d'Ailanthus glandulosa. Mémoire magister Univ. SBAbbes.

Treep L., 1975, Le choix des espèces en Algérie. Rapport 195 p.

\section{NOTES}

1. Les sols secs sont plus convenables pour sa croissance que les sols humides. Elle pousse même sur les sols sableux et sur une terre rocailleuse.

2. Avec un taux de germination maximale de $60 \%$ et une vitesse de germination de 63 jours qui sont des données remarquables comparées aux graines semées dans la zone tellienne (sachant que les graines de notre essai proviennent de cette région), avec un taux de germination de $63 \%$ et une vitesse de germination égale à 68 jours. L'ailante a enregistré une croissance en hauteur de $25 \mathrm{~cm}$ pour une période de 20 semaines.

L'accroissement moyen en hauteur est de $5 \mathrm{~cm}$ par mois, cet accroissement se traduit par une hauteur de l'ordre de $60 \mathrm{~cm}$ par an.

3. Il faut noter aussi que l'espèce a un pouvoir drageonnant très important, donc même si les conditions de culture sont défavorables provoquant le dessèchement de la plante mère, l'ailante donnera rapidement des rejets).

\section{RÉSUMÉS}

La steppe en Algérie est un espace stratégique en matière de lutte contre la désertification, il couvre plus de 20 millions d'hectares et connaît une dégradation importante. Différents programmes de protection et de réhabilitation de cet espace ont échoué et sont souvent à l'origine de l'aggravation de cette dégradation. Une synthèse sur les causes de cet échec permettront de proposer une nouvelle approche axée sur l'introduction d'une espèce végétale très rustique et dont l'impact écologique pourrait sauver la steppe.

The steppe in Algeria is a strategic space as regards to the control of desertification, it covers more than 20 million hectares and knows an important degradation. Various programmes of protection and rehabilitation concerning this space failed and are at the origin of the aggravation of this degradation. A synthesis of the causes of this failure will make it possible to propose a new approach centered on the introduction of a very rustic plant species and whose ecological impact could save the steppe.

\section{INDEX}

Keywords : dégradation, Ailanthus, impact, steppe, strategy, development Mots-clés : stratégie, développement 


\section{AUTEURS}

\section{MOHAMMEDI HALIMA}

Mohammedi Halima, Faculté des Sciences, université de Sidi Bel Abbes (Algérie)

\section{LABANI ABDERRAHMANE}

Labani Abderrahmane, LRSBG Centre universitaire de Mascara (Algérie)

\section{BENABDELI KHÉLOUFI}

Benabdeli Khéloufi, Centre Universitaire de Saida (Algérie) 